\title{
Long Term Cultures of Primary Human Hepatocytes as an Alternative to Drug Testing in Animals
}

\author{
Anett Ullrich ${ }^{1}$, Donna B. Stolz ${ }^{2}$, Ewa C. Ellis ${ }^{3}$, Stephen C. Strom ${ }^{3}$, George K. Michalopoulos ${ }^{3}$, \\ Jan G. Hengstler ${ }^{4}$ and Dieter Runge ${ }^{1}$ \\ ${ }^{1}$ PRIMACYT GmbH, Schwerin, Germany; ${ }^{2}$ University of Pittsburgh, Department of Cell Biology, Pittsburgh, USA; \\ ${ }^{3}$ University of Pittsburgh, Department of Pathology, Pittsburgh, USA; ${ }^{4}$ Leibniz Research Centre for Working Environment and \\ Human Factors, Dortmund, Germany
}

\begin{abstract}
Summary
Due to species differences, primary human hepatocytes are still the in vitro system of choice to analyse liver specific processes and functions. Human hepatocytes were cultured for several weeks in a serum-free twodimensional culture system, which was used to study the effects of acetaminophen (APAP) on hepatocellular functions and vitality. Non-invasive determinations of albumin, urea and lactate dehydrogenase concentrations in cell culture supernatants allowed continuous monitoring for at least two weeks. APAP was applied every 4 days for $24 \mathrm{~h}$. Each application reduced urea production by $25 \%$ and albumin synthesis by approximately $70 \%$ without any effects on cellular viability. After removal of the substance, hepatocellular functions returned to control levels within one (urea) to three (albumin) days. The repetitive analyses of APAP-mediated effects on cellular metabolism led to identical results for up to five cycles. The drug also caused reversible and repetitive ultrastructural modifications, in particular an almost complete replacement of rough endoplasmic reticulum by smooth endoplasmic reticulum and a massive degradation of glycogen stores. The data demonstrate the suitability of the culture system to serve as a model for repetitive testing of drug-mediated changes on hepatocellular functions, thereby reducing animal studies during drug development.
\end{abstract}

Keywords: human hepatocytes, acetaminophen, drug testing, animal studies

\section{Introduction}

In mammals the liver is the central organ for energy metabolism, synthesis of plasma proteins, detoxification of ammonia to urea and, last but not least, for metabolism and detoxification of endogenous and exogenous compounds. Although animal studies are necessary and required in preclinical development, in some cases clinical studies reveal toxic effects in humans, so that these substances need to be withdrawn. That is the major reason why we focused on use of primary human hepatocytes for the analyses of toxicity during preclinical studies. Today mostly primary hepatocytes from rat and mouse or hepatoma cell lines (e.g. HepG2) are used to study the influence of drugs in development on hepatocellular functions and viability and to analyse the in vitro metabolism of the compound. However, clear differences in cellular functions exist between the species, e.g. in CYP450 induction (Kocarek et al., 1995), in the intracellular distribution of gluconeogenic enzymes (Brech et al., 1970), in expression of apolipoproteins (Elshourbagy et al., 1986), and also in the metabolic regulation of cholesterol and triacylglycerol synthesis (Lin et al., 1995). In hepatoma cells, liver-specific proteins, like phase I and phase II enzymes, are expressed at significantly lower levels compared to primary hepatocytes (Bissell et al., 1973). Therefore, the cultivation of primary human hepatocytes exhibits the only system for a credible evaluation of xenobiotics in the context of preclinical studies. A major disadvantage is the lack of available material, which is mainly donated by patients with liver resections due to cancer. To counter this deficiency the long-term and serum-free cultivation of human hepatocytes was improved, mainly by adaptation of the culture medium (Runge et al., 1999; Runge et al., 2000; Ferrini et al., 1997). The generated HHMM (Human Hepatocyte Maintenance Medium) maintains human hepatocytes in a dif-

Received $25^{\text {th }}$ July 2009 , accepted for publication $8^{\text {th }}$ December 2009 
ferentiated state for 2-3 weeks as analysed by urea and albumin synthesis (Ullrich et al., 2007). We used this advantage to apply this two-dimensional long-term and serum-free human hepatocyte culture system to a repetitive screening of drugmediated changes. With acetaminophen as model substance, a commonly used antipyretic and analgesic compound was examined. More than $80 \%$ of the compound is metabolised by glucuronidation and sulfation (Josephy, 2005). The CYP450 proteins 3A4, 1A2 and 2E1 form the electrophile metabolite NAPQI (N-acetyl-p-benzoquinonimine), which is highly toxic (James et al., 2003). After overdose, cellular glutathione used for detoxification of NAPQI, is depleted, cellular functions are impaired by binding of NAPQI to macromolecules, which finally lead to centrilobular necrosis (Josephy, 2005).

\section{Materials and methods}

\section{Materials}

The basal media used for the preparation of HHMM and the ITS supplement were purchased from Invitrogen (Carlsbad, CA). The MEM medium was obtained from Sigma-Aldrich (St. Louis, MO). Epidermal Growth Factor (EGF) was supplied by BD Pharmingen (San Diego, CA); Hepatocyte Growth Factor (HGF, $\Delta 5$ variant) was donated by Snow Brand Co. (Toshigi, Japan). The antibodies for albumin ELISA were purchased from Bethyl Laboratories (Montgomery, TX). The urea assay kit was supplied by BioAssaySystems (Hayward, CA); the LDH cytotoxicity detection kit was purchased from Roche Diagnostics (Mannheim, Germany). The chemicals MTT (Methylthiazolyldiphenyl-tetrazolium bromide), acetaminophen, TMB (3,3',5,5'-Tetramethyl-benzidine) were purchased from Sigma-Aldrich (St. Louis, MO). General reagents for buffers and media supplements were obtained from Sigma-Aldrich.

\section{Isolation and culture of human hepatocytes}

Human hepatocytes were isolated from healthy tissue of liver resections due to cancer or from whole livers not acceptable for transplantation by three-step collagenase perfusion (Dorko et al., 1994). Liver specimens were taken with the informed consent of the donors. The study protocol has been approved by several ethics commissions: Ärztekammer Mecklenburg-Vorpommern, Ärztekammer Hamburg, University of Greifswald, University of Rostock, and the University of Kiel. The viability of cells was determined by trypan blue exclusion test, and only hepatocyte preparations with a viability higher than $70 \%$ were used in the experiments. Cells were plated onto collagen-coated 6-well plates (UV-dried rat tail collagen solution $0.2 \mathrm{mg} /$ $\mathrm{ml}$ ) at a density of 100,000 cells $/ \mathrm{cm}^{2}$ using MEM (Minimal Essential Medium) containing $500 \mathrm{ng} / \mathrm{ml}$ insulin and $50 \mu \mathrm{g} /$ $\mathrm{ml}$ gentamycin. Usually the medium was changed after 3-12 $\mathrm{h}$ to HHMM (Human Hepatocyte Maintenance Medium, Primacyt $\mathrm{GmbH}$, Germany) containing $10 \mathrm{ng} / \mathrm{ml} \mathrm{HGF}$ and $20 \mathrm{ng} / \mathrm{ml}$ EGF. The medium was changed daily during culture ( $24 \mathrm{~h}$ cycle). The high cell density was chosen because the expression of CYP450 proteins and other liver specific functions depend more on cell-cell than on cell-matrix contacts (Hamilton et al., 2001; Isom et al., 1984).

\section{Application of acetaminophen}

Acetaminophen (APAP) was dissolved in HHMM with $40 \%$ DMSO to obtain a stock solution of $0.1 \mathrm{~g} / \mathrm{ml}$. The test substance was used at a final concentration of $2815 \mathrm{mg} / \mathrm{l}(=18.6$ $\mathrm{mM}$ ) and 1.12-1.6\% DMSO as a solvent control. The concentration of APAP used for this study represents the $\mathrm{IC}_{50}$-value determined with multiple toxicity tests on primary cells and cell lines (Clemedson et al., 1996). The identical concentration was used in a preceding publication (Ullrich et al., 2007), where we first described the culture system. After adaptation of human hepatocytes to the in vitro conditions during the first 3 days, the incubation with APAP was started on day 4 of culture for $24 \mathrm{~h}$. The medium without APAP was renewed daily by HHMM until day 8 when the second application cycle was initiated. Further incubations with APAP were performed on day 12 and day 16 , for $24 \mathrm{~h}$ each.

\section{Biochemical assays \\ - Albumin ELISA}

Albumin release into culture medium was determined using a sandwich ELISA with antibodies obtained from Bethyl Laboratories (Montgomery, TX). For quantification of human albumin, goat anti-human albumin antibody (1:200) was used for coating and goat anti-human albumin HRP (horseradish peroxidase) conjugated antibody $(1: 25,000)$ was used for detection of bound albumin. Quantification was possible with diluted standard samples of human reference serum with a defined albumin concentration. The assay was performed according to the manufacturer's instructions with high-binding ELISA 96-well plates obtained from Greiner BioOne (Monroe, NC). Bound HRP antibodies were detected with TMB (SigmaAldrich, St. Louis, MO) for $15 \mathrm{~min}$, the reaction was stopped with $0.5 \mathrm{M} \mathrm{H}_{2} \mathrm{SO}_{4}$ and quantified by measuring the absorbance at $450 \mathrm{~nm}$.

\section{- Urea assay}

The method for quantification of urea in cell culture supernatants is based on reactions described by Jung et al. (1975). Primaquinphosphate was used as the colour reagent instead of NED (N-(1-Naphthyl)ethylendiamine). The optical density was detected at $520 \mathrm{~nm}$ as required in the detailed protocol provided by the manufacturer.

\section{- LDH assay}

In this assay the LDH enzyme catalyses the oxidation of lactate to pyruvate with reduction of $\mathrm{NAD}^{+}$. In a second step, the enzyme diaphorase converts the yellow tetrazolium salt INT (Iodophenyl-nitrophenyl-phenyltetrazoliumchloride) to the red formazan with oxidation of $\mathrm{NADH}^{+} \mathrm{H}^{+}$. Formazan shows a maximum absorption at $490 \mathrm{~nm}$, which correlates directly with LDH activity. The activity was quantified with standard dilutions of hog muscle L-LDH. 


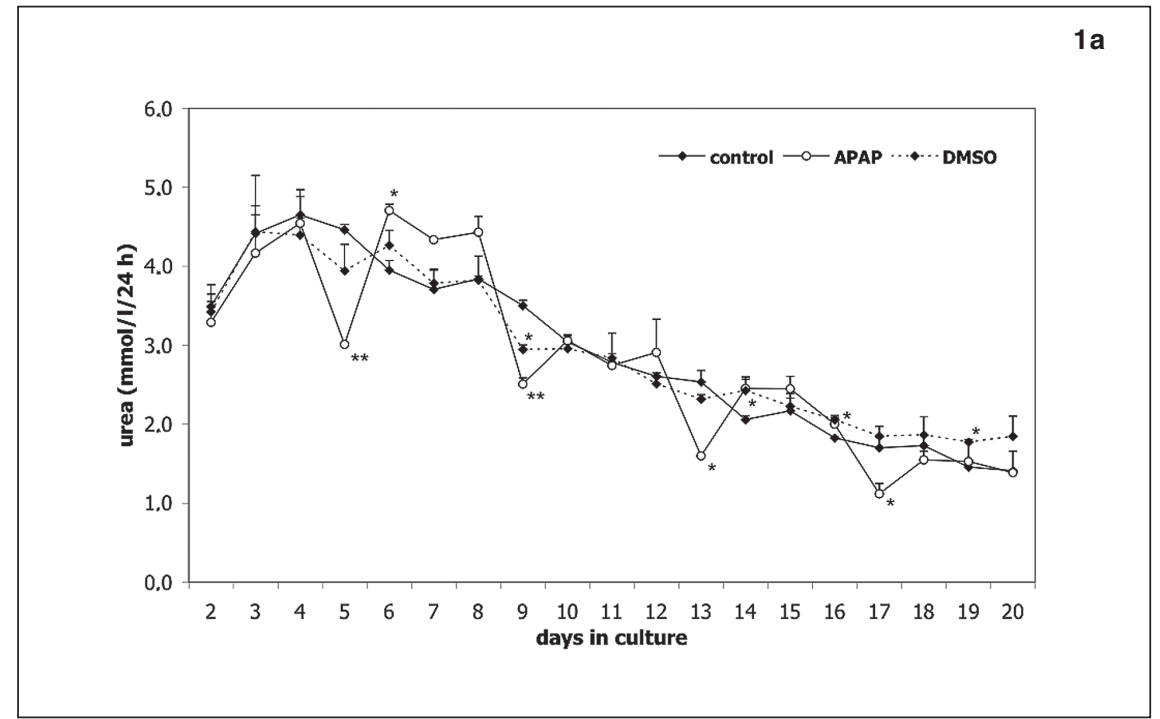

Fig. 1: Acetaminophen causes a repeated and reversible impact on hepatocellular functions, but not on cell viability.

1a: urea release $(\mathrm{mmol} / / / 24 \mathrm{~h}) ; 1 \mathrm{~b}$ : albumin release $(\mathrm{mg} / \mathrm{l} / 24 \mathrm{~h})$; 1c: LDH activity $\left(\mu \mathrm{mol} / /^{*} \mathrm{~s} / 24 \mathrm{~h}\right)$. Human hepatocytes were cultured in HHMM with HGF and EGF; medium was changed daily. APAP (2815 $\mathrm{mg} / \mathrm{l})$ or DMSO (1.6\%) was applied on days 4, 8, 12 and 16 for $24 \mathrm{~h}$ each. Statistics (Student's t-test): ${ }^{*}=p<0.05,{ }^{* *}=p<0.01$; the statistical differences were calculated with the APAP or DMSO values compared to controls of the same day. Data of hepatocytes from one representative donor out of 7 are shown.

$1 b$

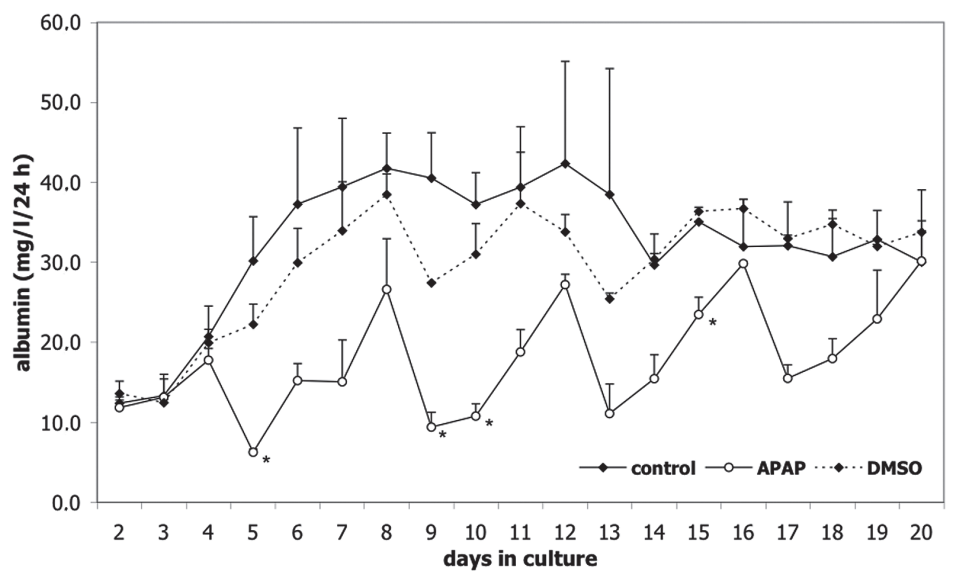

$1 \mathrm{c}$

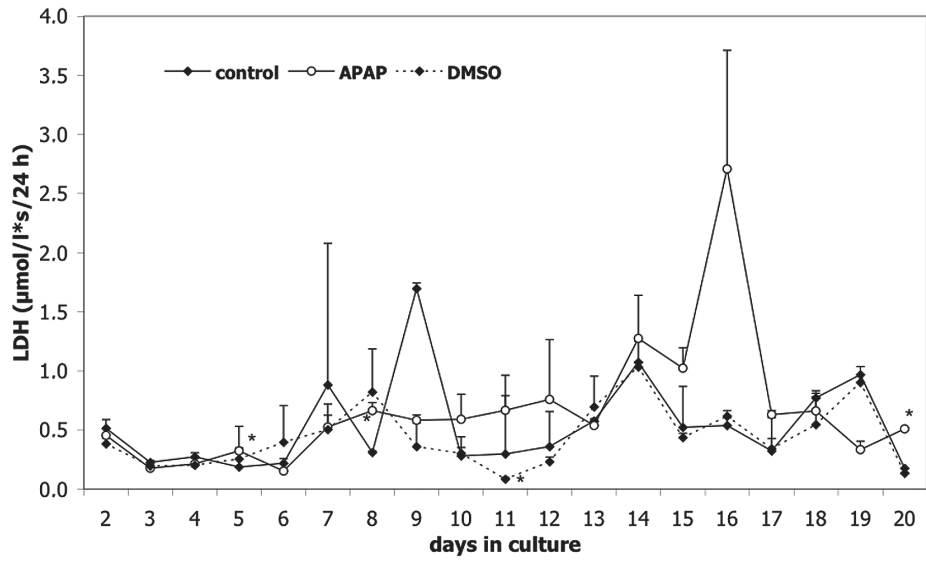


Transmission electron microscopy (TEM)

Monolayers in 6-well plates used for electron microscopy were washed with PBS twice and fixed in $2.5 \%$ glutaraldehyde for 2 $\mathrm{h}$ at $4^{\circ} \mathrm{C}$. The further procedure was done as described in Michalopoulos et al. (1999). Electron micrographs with hepatocytes from two human donors were examined in duplicates for each treatment to study the ultrastructural changes caused by acetaminophen.

\section{Results and discussion}

\section{Heterogeneity of the donors}

Primary human hepatocytes are as heterogeneous as their donors, especially in the proportion of different CYP450 enzymes and their activities (Gomez-Lechon et al., 2004). Also, the metabolic activities as detected by the amount of urea and albumin in culture medium varied between the cultures. In Figure 1a-c hepatocyte specific data of one exemplary culture out of 7 is presented to give absolute data. The Table 1a-c shows the summarised results of 7 individual experiments with hepatocytes from 7 different donors.

\section{Effect of acetaminophen on hepatocellular functions}

The two-dimensional human hepatocyte culture system with the chemically defined, serum-free HHMM keeps the cells in a highly differentiated state for at least two weeks, and the growth factors HGF and EGF help to maintain these functions (Ullrich et al., 2007). This system was used to analyse drugrelated changes in hepatocellular function and morphology.

The detoxification of ammonia to urea in human hepatocytes was diminished to $70 \%$ of the untreated/DMSO-treated cells during the first application of APAP from day 4 to day 5 of culture. Already $24 \mathrm{~h}$ after removal of the active substance and its metabolites, the urea concentration in cell culture supernatants returned to control levels. This reduction of urea release occurred during each application cycle. No matter whether the substance was applied early in culture or on day 16 of culture, the influence of APAP on ammonia metabolism and urea production was nearly identical. In every one of the four cycles shown in Figure 1a, APAP led to a similar decrease in urea release to $60-75 \%$ of control levels.

The impact of APAP $(18.6 \mathrm{mM})$ on the synthesis of the plasma protein albumin was much greater than on urea production. The albumin production and release from the cells decreased to $30 \%$ of control values (negative and solvent control). Due to the larger influence of this hepatocellular function, it took $72 \mathrm{~h}$ until human hepatocytes reached the albumin secretion rate of control cells. As already shown for urea synthesis (Fig. 1a), the effect on albumin synthesis and release found in the first cycle from day 4 to day 5 was reproducible at later time points in the culture, i.e. on days 8,12 and 16, including a recovery period of 3 days (Fig. 1b).

The reduction of anabolic and catabolic functions shown in Figure $1 \mathrm{a}$ and $\mathrm{b}$ were not caused by lower viability of human hepatocytes as a result of APAP-treatment for $24 \mathrm{~h}$. Only dur- ing the third cycle, starting on day 12, a small, but non-significant increase in $\mathrm{LDH}$ release was detected on day 16, i.e. not during, but three days after the application of APAP (Fig. 1c). In addition, the APAP-treated cells did not show any morphologic changes/abnormalities to solvent control cells observed by phase-contrast light microscopy. The human hepatocytes in both approaches showed mononuclear, vital cells with a typical polygonal shape (micrographs not shown). A concentration of $2815 \mathrm{mg} / \mathrm{l}$ APAP did not lead to a depletion of glutathione when incubated with hepatocytes for $24 \mathrm{~h}$ (data not shown).

Furthermore, concentrations higher than and below 2815 $\mathrm{mg} / \mathrm{l}$ were also examined. Tested concentrations of $100 \mathrm{mg} / \mathrm{l}$ and $500 \mathrm{mg} / \mathrm{l}$ APAP did not show any significant effects on albumin and urea synthesis. Higher single doses of $4000 \mathrm{mg} / \mathrm{l}$ APAP led to a $60 \%$ decrease in urea release and to an $87 \%$ reduction of albumin synthesis in primary human hepatocytes. These cells were able to recover only one metabolic function completely. The urea synthesis rate returned to control level within $24 \mathrm{~h}$, whereas the amount of synthesised albumin did not reach the value of control cells within 3 days after removal of the active substance and its metabolic products (data not shown).

\section{Ultrastructural changes due to APAP}

Moreover we examined how far the effects of APAP on hepatocellular functions go along with ultrastructural changes. Also, their reversibility and reproducibility were analysed by electron microscopy. Before the first application of APAP was started on day 4, hepatocytes contained high amounts of glucose stored as glycogen in the cells. Polyribosomes and almost exclusively rough endoplasmic reticulum (ER) were found, two signs for a distinct rate of protein synthesis. The proportion of smooth ER was very low. Hepatocytes treated with $2815 \mathrm{mg} / \mathrm{ml}$ (18.6 mM) APAP for $24 \mathrm{~h}$ showed predominantly smooth ER, and the glycogen stores were completely degraded on day 5. This complete switch from rough to smooth ER and glycogen catabolism was not found in cells treated with the solvent control DMSO. Already within one day after removal of the substance the cells were able to refill their glycogen stores. The ratio of rough to smooth ER was still way in favour of smooth ER as an organelle for biotransformation of endogenous and exogenous compounds on day 6. Not until day 7 did the proportion of rough ER prevail over smooth ER. Human hepatocytes are able to regenerate from ultrastructural modifications within two days after removal of the substance (Fig. 2). Furthermore, it was analysed whether ultrastructural changes observed within the first application of APAP also occur after multiple cycles of xenobiotic treatment. In the $3^{\text {rd }}$ (started on day 12) and in the $5^{\text {th }}$ (started on day 20) cycle of APAP application human hepatocytes showed nearly identical modifications in ultrastructure as in the first cycle. The glycogen stores were also abolished completely. The decline of rough ER was not as massive as in the first cycle from day 4 to day 5, but much smooth ER was still detected in much higher amounts than in control cultures. 
Tab. 1a: Urea release in human hepatocytes (in \%)

\begin{tabular}{|llllll|}
\hline 1. cycle & day $\mathbf{4}$ & day $\mathbf{5}$ & day $\mathbf{6}$ & day $\mathbf{7}$ & day $\mathbf{8}$ \\
\hline control & $100 \pm 7$ & $78 \pm 34$ & $92 \pm 8$ & $87 \pm 11$ & $82 \pm 11$ \\
APAP & $97 \pm 8$ & $68 \pm 12$ & $102 \pm 11$ & $100 \pm 11$ & $96 \pm 12$ \\
DMSO & $99 \pm 7$ & $92 \pm 17$ & $96 \pm 1^{* \star *}$ & $86 \pm 5$ & $78 \pm 8$ \\
2. cycle & day $\mathbf{8}$ & day $\mathbf{9}$ & day $\mathbf{1 0}$ & day $\mathbf{1 1}$ & day $\mathbf{1 2}$ \\
\hline control & $100 \pm 10$ & $93 \pm 5$ & $86 \pm 12$ & $77 \pm 13$ & $76 \pm 15$ \\
APAP & $109 \pm 17$ & $62 \pm 10^{* * *}$ & $89 \pm 14$ & $81 \pm 13$ & $82 \pm 16$ \\
DMSO & $104 \pm 12$ & $80 \pm 3^{* *}$ & $85 \pm 15$ & $84 \pm 13$ & $70 \pm 6$ \\
3. cycle & day $\mathbf{1 2}$ & day 13 & day $\mathbf{1 4}$ & day 15 & day 16 \\
\hline control & $100 \pm 8$ & $91 \pm 12$ & $85 \pm 10$ & $84 \pm 8$ & $80 \pm 9$ \\
APAP & $117 \pm 19$ & $63 \pm 8^{* * *}$ & $94 \pm 12$ & $90 \pm 8$ & $75 \pm 10$ \\
DMSO & $121 \pm 45$ & $86 \pm 7$ & $92 \pm 12$ & $88 \pm 5$ & $89 \pm 6$ \\
4. cycle & day $\mathbf{1 6}$ & day $\mathbf{1 7}$ & day $\mathbf{1 8}$ & day 19 & day 20 \\
\hline control & $100 \pm 8$ & $100 \pm 10$ & $90 \pm 11$ & $90 \pm 7$ & $86 \pm 14$ \\
APAP & $110 \pm 12^{*}$ & $73 \pm 26^{*}$ & $95 \pm 17$ & $89 \pm 15$ & $82 \pm 19$ \\
DMSO & $141 \pm 40$ & $84 \pm 16$ & $85 \pm 10$ & $85 \pm 5$ & $81 \pm 12$ \\
\hline
\end{tabular}

Tab. 1b: Albumin release in human hepatocytes (in \%)

\begin{tabular}{|c|c|c|c|c|c|}
\hline 1. cycle & day 4 & day 5 & day 6 & day 7 & day 8 \\
\hline control & $100 \pm 13$ & $142 \pm 24$ & $192 \pm 43$ & $203 \pm 46$ & $238 \pm 71$ \\
\hline APAP & $131 \pm 69$ & $29 \pm 9^{* * *}$ & $74 \pm 15^{\star \star \star}$ & $122 \pm 0^{\star \star *}$ & $169 \pm 46$ \\
\hline DMSO & $112 \pm 31$ & $102 \pm 13^{*}$ & $149 \pm 3^{*}$ & $180 \pm 19$ & $218 \pm 26$ \\
\hline 2. cycle & day 8 & day 9 & day 10 & day 11 & day 12 \\
\hline control & $100 \pm 18$ & $96 \pm 25$ & $100 \pm 23$ & $101 \pm 26$ & $129 \pm 35$ \\
\hline APAP & $90 \pm 22$ & $25 \pm 11^{* \star *}$ & $49 \pm 17^{* *}$ & $88 \pm 33$ & $130 \pm 47$ \\
\hline DMSO & $85 \pm 33$ & $88 \pm 19$ & $100 \pm 36$ & $115 \pm 15^{*}$ & $115 \pm 25$ \\
\hline 3. cycle & day 12 & day 13 & day 14 & day 15 & day 16 \\
\hline control & $100 \pm 21$ & $94 \pm 23$ & $88 \pm 31$ & $106 \pm 38$ & $103 \pm 55$ \\
\hline APAP & $95 \pm 33$ & $28 \pm 13^{\star \star \star}$ & $47 \pm 17^{*}$ & $82 \pm 40$ & $129 \pm 47$ \\
\hline DMSO & $103 \pm 65$ & $96 \pm 21$ & $95 \pm 5$ & $109 \pm 21$ & $104 \pm 8$ \\
\hline 4. cycle & day 16 & day 17 & day 18 & day 19 & day 20 \\
\hline control & $100 \pm 12$ & $105 \pm 14$ & $106 \pm 9$ & $107 \pm 16$ & $95 \pm 19$ \\
\hline APAP & $117 \pm 61$ & $27 \pm 13^{\star \star \star}$ & $39 \pm 12^{\star \star \star}$ & $64 \pm 19^{\star \star \star}$ & $87 \pm 19$ \\
\hline DMSO & $116 \pm 52$ & $95 \pm 10$ & $87 \pm 19$ & $88 \pm 8^{*}$ & $85 \pm 10$ \\
\hline
\end{tabular}

Tab. 1c: LDH activity in human hepatocytes (in \%)

\begin{tabular}{|c|c|c|c|c|c|}
\hline 1. cycle & day 4 & day 5 & day 6 & day 7 & day 8 \\
\hline control & $100 \pm 14$ & $78 \pm 40$ & $75 \pm 58$ & $130 \pm 118$ & $94 \pm 38$ \\
\hline APAP & $91 \pm 33$ & $109 \pm 112$ & $125 \pm 81$ & $152 \pm 68$ & $167 \pm 103$ \\
\hline DMSO & $115 \pm 38$ & $129 \pm 4^{*}$ & $134 \pm 89$ & $151 \pm 140$ & $228 \pm 254$ \\
\hline 2. cycle & day 8 & day 9 & day 10 & day 11 & day 12 \\
\hline control & $100 \pm 8$ & $189 \pm 177$ & $102 \pm 19$ & $107 \pm 20$ & $149 \pm 37$ \\
\hline APAP & $135 \pm 68$ & $134 \pm 234$ & $105 \pm 49$ & $96 \pm 30$ & $98 \pm 38$ \\
\hline DMSO & $130 \pm 91$ & $162 \pm 154$ & $99 \pm 63$ & $65 \pm 50$ & $75 \pm 66$ \\
\hline 3. cycle & day 12 & day 13 & day 14 & day 15 & day 16 \\
\hline control & $100 \pm 17$ & $103 \pm 54$ & $134 \pm 99$ & $93 \pm 54$ & $94 \pm 52$ \\
\hline APAP & $95 \pm 58$ & $37 \pm 36^{*}$ & $117 \pm 74$ & $123 \pm 77$ & $167 \pm 133$ \\
\hline DMSO & $76 \pm 32$ & $169 \pm 186$ & $240 \pm 292$ & $100 \pm 124$ & $141 \pm 179$ \\
\hline 4. cycle & day 16 & day 17 & day 18 & day 19 & day 20 \\
\hline control & $100 \pm 12$ & $97 \pm 32$ & $120 \pm 31$ & $125 \pm 54$ & $87 \pm 39$ \\
\hline APAP & $199 \pm 178$ & $79 \pm 111$ & $73 \pm 38^{*}$ & $86 \pm 43$ & $81 \pm 56$ \\
\hline DMSO & $100 \pm 41$ & $144 \pm 77$ & $113 \pm 26$ & $111 \pm 45$ & $44 \pm 31$ \\
\hline
\end{tabular}

Tab. 1: Influence of acetaminophen on hepatocellular functions and vitality summary of 7 different donors.

Human hepatocytes were cultured in HHMM with HGF and EGF; medium was changed daily. APAP $(2815 \mathrm{mg} / \mathrm{l})$ or DMSO (1.12-1.6\%) was applied on days 4, 8,12 and 16 for $24 \mathrm{~h}$ each. The amount of urea and albumin synthesised and $\mathrm{LDH}$ released in $24 \mathrm{~h}$ before start of the application were set as $100 \%$. The data from the following 4 days were calculated as a percentage of that of the application day. The tables represent means \pm SD of 7 cultures with hepatocytes of 7 different donors. The synthesis and release of urea was reduced by APAP by $25-40 \%$ and returned to control levels within $24 \mathrm{~h}$

(Tab. 1a). Albumin production and release was reduced by APAP to $70 \%$ and returned to control levels within $48 \mathrm{~h}$ (Tab. 1b). The treatment with APAP did not show any significant influence on the viability of human hepatocytes from 7 different donors (Tab. 1c).

Statistics (Student's t-test): ${ }^{*}=p<0.05$, ${ }^{* *}=p<0.01,{ }^{* * *}=p<0.001$;

the statistical differences were calculated with the APAP or DMSO values compared to controls of the same day. 
Even after recovery of the cells from the $5^{\text {th }}$ treatment with APAP, no differences between APAP and DMSO-treated human hepatocytes were found.

Therefore, human hepatocytes cultured serum-free under optimal conditions retain their hepatocyte-specific functions measured as synthesis of urea and albumin. These differentiated hepatocytes may be used for multiple cycles of drug testing, as shown here with APAP as a model substance.

The results demonstrate the ability of human hepatocytes to compensate functional as well as ultrastructural changes due to APAP within 48-72 $\mathrm{h}$ after removal of the compound. Due to this fact, one human hepatocyte culture may be used multiple times for toxicity testing of xenobiotics. In case of nontoxic effects of the compound, the same cells can metabolise a second compound after a recovery period of 3-4 days. Hepatocellular functions are monitored by non-invasive detection of albumin and urea secreted into the cell culture medium. The key benefits in using this method during preclinical development are: i) evaluation of toxicity on human hepatocytes gives more accurate results and eliminates the extrapolation from studies with animal hepatocytes; ii) animal studies are reduced; iii) the rare human liver tissue can be used optimally. The robustness of the culture system as shown here with the repetitive treatment with APAP favours it as an alternative method to the 28 days toxicity feeding study in animals.

Results from in vivo studies in animals and humans are needed to validate the system introduced here as an alternative method for animal studies. In a study published recently, the potential toxicity of the anticancer drug cis-4-hydroxy-proline (CHP) was tested in animal studies and in the human hepatocyte culture system described here (Dickens et al., 2008). The data obtained in both experiments were compared with data

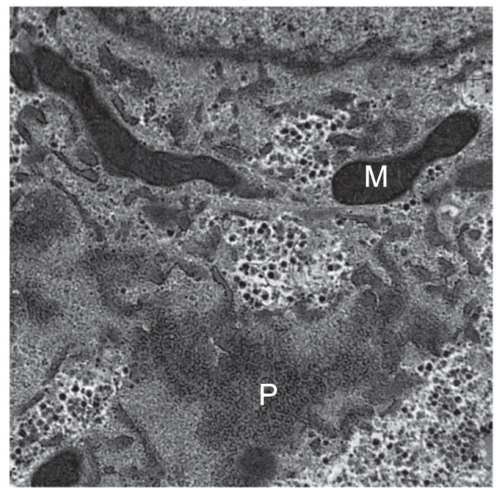

day 4 control $(4000 x)$

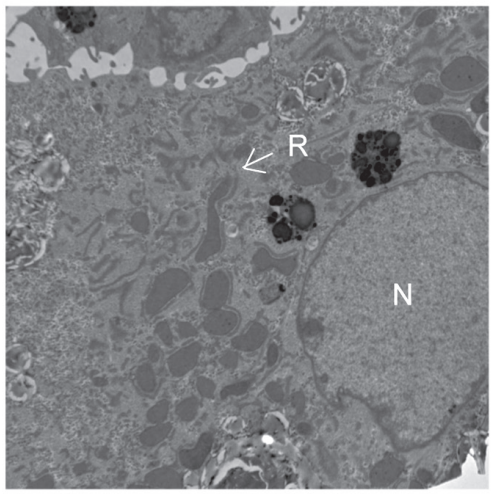

day 5 control (1500x)

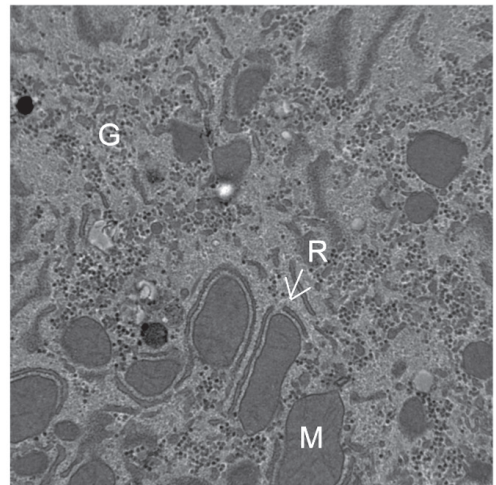

day 5 DMSO (3000x)

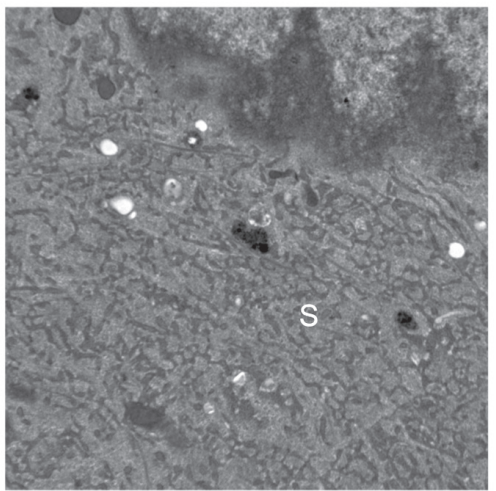

day 5 APAP (2500x)

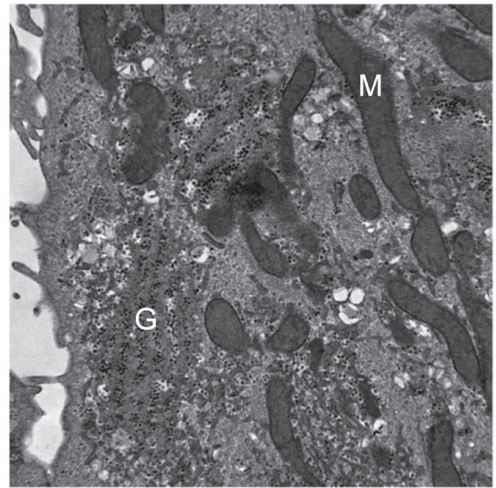

day 6 APAP (3000x)

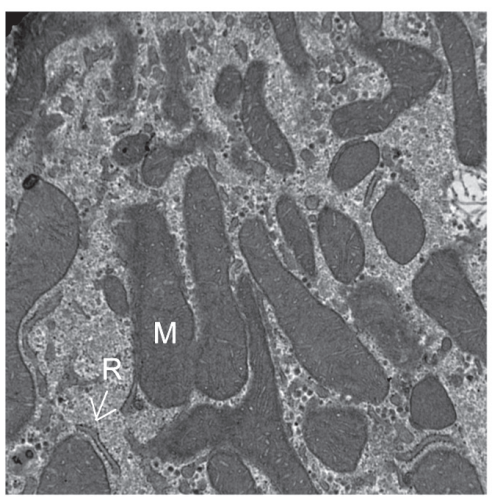

day 7 APAP (4000x)

Fig. 2: Acetaminophen causes ultrastructural changes that are reversible and repeatedly inducible at later application cycles. Human hepatocytes were cultured in HHMM with HGF and EGF; medium was changed daily. The application of APAP (2815 mg/l) or DMSO (1.12\%) was carried out from day 4 every 4 days for $24 \mathrm{~h}$ each treatment. At the indicated time points cells were fixed with $2 \%$ paraformaldehyde for processing in TEM; abbreviations: $\mathrm{G}$ - glycogen, R - rough ER, S - smooth ER, P - polyribosomes, $\mathrm{N}$ - nucleus, $\mathrm{M}$ - mitochondria, $\mathrm{F}$ - fat droplets.

2a: First cycle with APAP treatment from day 4 to day 5

$2 \mathrm{~b}$ : Fifth cycle with APAP treatment from day 20 to day 21, the addition of APAP $(2815 \mathrm{mg} / \mathrm{l})$ was carried out for $24 \mathrm{~h}$ beginning on day 4 of culture on every $4^{\text {th }}$ day. The micrograph of day 20 APAP shows cells which were already treated with APAP on days $4,8,12$ and 16 for $24 \mathrm{~h}$ each. 
obtained in a phase II study, where the toxic harmlessness of the compound in humans was demonstrated. While the animal studies would have led to the wrong conclusion of a defined toxic potential of the compound, the in vitro study confirmed the toxic harmlessness of the substance in humans.

For an acceptance as a replacement of animal studies, an evaluation with a much higher number of non-toxic/toxic substances needs to be done. The most important point the authors want to demonstrate with this article is the robustness of the human hepatocyte culture system, allowing the use of the same culture for multiple drug tests, leading to the same results each time. Also, preliminary data obtained in studies in which two substances that have different (opposite) effects on urea and albumin release are given alternately showed that their impact on hepatocellular functions were not altered, no matter which substance was given first. The data presented here give a first indication that the system may be a useful model that could lead to a reduction of 28 day toxicity animal studies. Due to its robustness the system qualifies for further studies with substances that are toxic upon repeated administration.

\section{References}

Bissell, D. M., Hammaker, L. E. and Meyer, U. A. (1973). Parenchymal cells from adult rat liver in nonproliferating monolayer culture - I. Functional studies. J. Cell Biol. 59, 722-734.

Brech, W., Shrago, E. and Wilken, D. (1970). Studies on pyruvate carboxylase in rat and human liver. Biochim. Biophyss. Acta. 201, 145-154.

Clemedson, C., McFarlane-Abdulla, E., Andersson, M. et al.
$2 b$

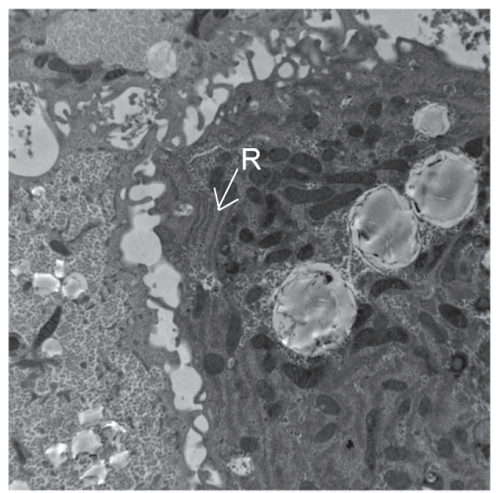

day 20 control (2500x)

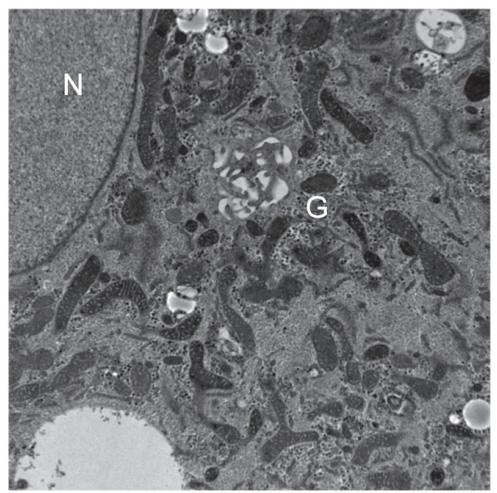

day $20 \operatorname{APAP}(2500 x)$

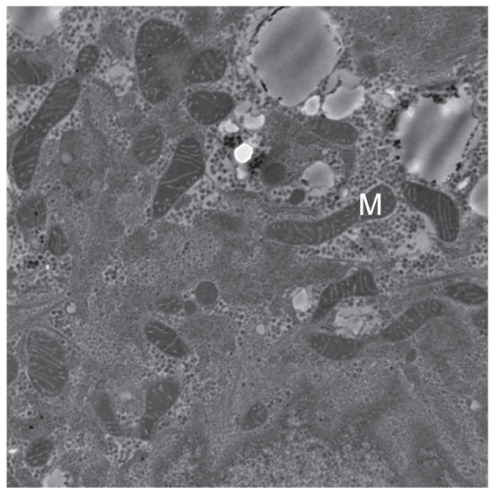

day 21 control (4000x)

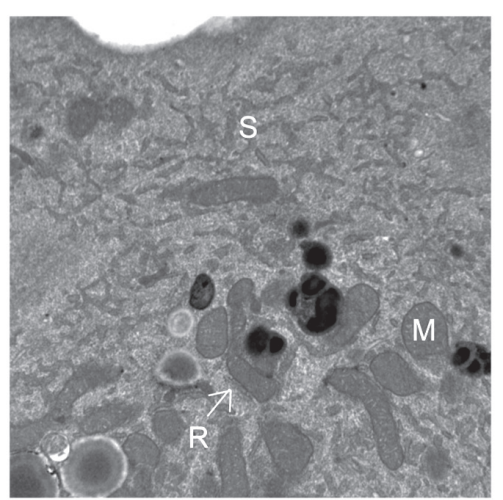

day 21 APAP (4000x)

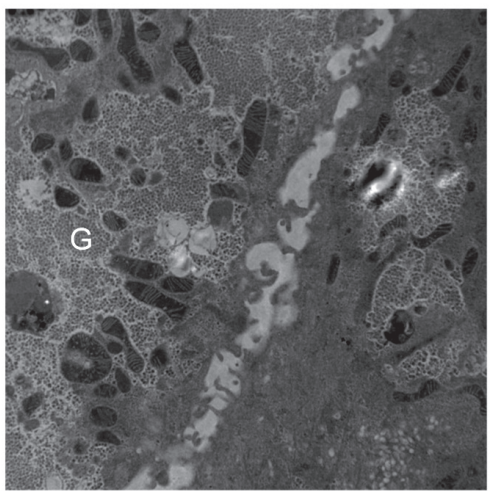

day 22 control (2500x)

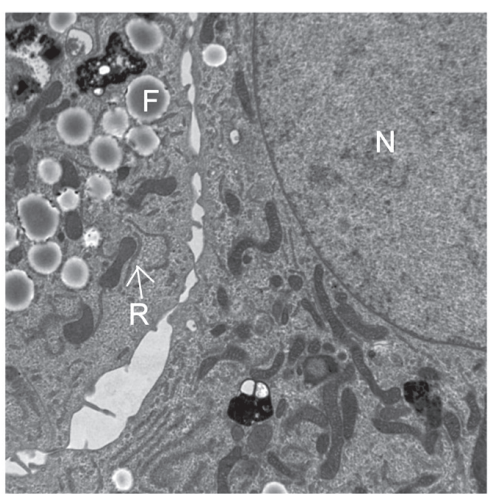

day $22 \operatorname{APAP}(2500 x)$ 
(1996). MEIC Evaluation of acute systemic toxicity - Part II: In vitro results from 68 toxicity assays used to test the first 30 reference chemicals and a comparative cytotoxicity analysis. ATLA 24, 273-311.

Dickens, H., Ullrich, A., Runge, D. et al. (2008). Anticancer drug cis-4-hydroxy-L-proline: Correlation of preclinical toxicology with clinical parameters of liver function. Mol. Med. Reports 1, 459-464.

Dorko, K., Freeswick, P. D., Bartoli, F. et al. (1994). A new technique for isolating and culturing human hepatocytes from whole or split livers not used for transplantation. Cell Transplant. 3, 387-395.

Elshourbagy, N. A., Walker, D. W., Boguski, M. S. et al. (1986). The nucleotide and derived amino acid sequence of human apolipoprotein A-IV mRNA and the close linkage of its gene to the genes of apoplipoproteins A-I and C-III. J. Biol. Chem. 261, 1998-2002.

Ferrini, J. B., Pichard, L., Domerque, J. and Maurel, P. (1997). Long-term primary cultures of adult human hepatocytes. Chem. Biol. Interact. 107, 31-45.

Gomez-Lechon, M. J., Donato, M. T., Castell, J. V. and Jover, R. (2004). Human hepatocytes in primary culture: The choice to investigate drug metabolism in man. Curr. Drug Metab. 5 , 443-462.

Hamilton, G. A., Jolley, S. L., Gilbert, D. et al. (2001). Regulation of cell morphology and cytochrome P450 expression in human hepatocytes by extracellular matrix and cell-cell interactions. Cell Tissue Res. 306, 85-99.

Isom, H. C., Secott, T., Georgoff, I. et al. (1984). Maintenance of differentiated rat hepatocytes in primary culture. Proc. Natl. Acad. Sci. USA 82, 3252-3256.

James, L. P., Mayeux, P. R. and Hinson, J. A. (2003). Acetaminophen-induced hepatotoxicity. Drug Metab. Dispos. 31, 1499-1506.

Josephy, P. D. (2005). The molecular toxicology of acetaminophen. Drug Metab. Rev. 37, 581-594.

Jung, D., Biggs, H., Erikson, J. and Ledyard, P. U. (1975). New colorimetric reaction for end-point, continuous-flow, and kinetic measurement of urea. Clin. Chem. 21, 1136-1140.

Kocarek, T. A., Schuetz, E. G., Strom, S. C. et al. (1995). Comparative analysis of cytochrome $\mathrm{P} 4503 \mathrm{~A}$ induction in primary cultures of rat, rabbit, and human hepatocytes. Drug Metab. Dispos. 23, 415-421.

Lin, Y., Vonk, R. J., Slooff, M. J. et al. (1995). Differences in propionate-induced inhibition of cholesterol and triacylglycerol synthesis between human and rat hepatocytes in primary culture. Br. J. Nutr. 74, 197-207.

Michalopoulos, G. K., Bowen, W. C., Zajac, V. F. et al. (1999). Morphogenetic events in mixed cultures of rat hepatocytes and nonparenchymal cells maintained in biological matrices in the presence of hepatocyte growth factor and epidermal growth factor. Hepatology 29, 90-100.

Runge, D., Köhler, C., Kostrubsky, V. E. et al. (2000). Induction of Cytochrome P450 (CYP)1A1, CYP1A2, and CYP3A4 but not CYP2C9, CYP2C19, multidrug resistance (MDR-1) and multidrug resistance associated protein (MRP-1) by prototypical inducers in human hepatocytes. Biochem. Biophys. Res. Commun. 273, 333-341.

Runge, D. M., Runge, D., Foth, H. et al. (1999). STAT1 $\alpha / 1 \beta$, STAT3 and STAT5: Expression and association with c-MET and EGF-receptor in long-term cultures of human hepatocytes. Biochem. Biophys. Res. Commun. 265, 376-381.

Ullrich, A., Berg, C., Hengstler, J. G. and Runge, D. (2007). Use of a standardized and validated long-term human hepatocyte culture system for repetitive analyses of drugs: Repeated administrations of Acetaminophen reduces albumin and urea secretion. ALTEX 24, 35-40.

\section{Acknowledgements}

This work was supported in part by the State of MecklenburgVorpommern, grant V 230-630.08-TIFA-373 assigned to Dieter Runge.

\section{Correspondence to}

Anett Ullrich

PRIMACYT Cell Culture Technology GmbH

Hagenower Str. 73

19061 Schwerin, Germany

Phone: +49-385-3993600

Fax: +49-385-3993602

e-mail: anett.ullrich@primacyt.de 